\title{
Cyanobakterienkultivierung zur Extraktion toxischer Metabolite
}

\author{
Michél Hellmann, Judith Offermann, Franz Wildenauer
}

\section{Zusammenfassung}

Im Rahmen des Projektes »CyToTest « soll, in Kooperation mit der Firma BioTeZ Berlin-Buch GmbH, ein System entwickelt werden, mit dem direkt am Gewässer auf einfache Art und Weise die Cyanobakterientoxinkonzentration ermittelt werden kann. Dazu ist es nötig Cyanobakterien zu kultivieren und die Toxine zu isolieren. Beides wird gebraucht, um einerseits das Messsystem entwickeln zu können und andererseits an der TFH Wildau ein Verfahren zu entwickeln, mit dem die Cyanobakterien direkt am Gewässer aufgeschlossen und die Toxine extrahiert werden können. Im Rahmen dessen wurden sechs Gattungen Cyanobakterien kultiviert und ein Verfahren etabliert, um die Biomasse kalkulieren zu können. Weiterhin wurde mit der Kalibration der HPLC mit Toxinen begonnen, um die Extrakte später prüfen zu können.

\begin{abstract}
Within the project (in cooperation with BioTeZ BerlinBuch GmbH) »CyToTest « a system shall be developed which allows to detect the concentration of cyanotoxins directly at the waterbody in a simple way. Therefore it is necessary to cultivate cyanobacteria and to isolate their toxins. This is essential for developing the measuring system and a procedure for digest the cyanobacteria and for extract the toxins directly at the waterbody. Therefore six species of cyanobacteria were cultivated and a method was developed for calculating the biomass. Furthermore the calibration of the HPLC with the toxins was begun for checking the extracts.
\end{abstract}

\section{$1 \quad$ Einleitung}

Mit der am 15.02.2006 veröffentlichten EU-Badegewässerrichtlinie wird sich der Aufwand zur Überwachung der Gewässer deutlich erhöhen. Die neue Richtlinie schreibt vor, dass alle potentiellen Badegewässer als solche deklariert werden müssen. Die Richtlinie gilt für jeden Bereich eines Badegewässers.

Neu aufgenommen wurde ein Artikel zur Gefährdung durch Cyanobakterien: »Deutet das Profil des Badegewässers auf ein Potenzial für eine Massenvermehrung von Cyanobakterien hin, so wird eine geeignete Überwachung durchgeführt, damit Gefahren für die Gesundheit rechtzeitig erkannt werden können.«

Für jedes Gewässer muss ein Badegewässerprofil angelegt und ständig aktualisiert werden. In diesem ist u. a. eine Bewertung der Gefahr der Massenvermehrung von Cyanobakterien vorzunehmen. Pro Badesaison muss jedes Badegewässer mindestens viermal getestet werden. Für jeden Datensatz werden mindestens 16 Proben genommen. Der Zeitraum zwischen den Probennahmen darf einen Monat auf keinen Fall überschreiten. Die Analyse der entnommenen Probe sollte laut Richtlinie so schnell wie möglich erfolgen. Die Richtlinie gilt für den gesamten EU-Raum mit seinen einigen hunderttausend Seen und mit seinen tausenden Küstenkilometern.

In der Richtlinie wurde auch eine ständige Anpassung an den Fortschritt, vor allem der Technik zur Analyse der Wasserqualität, beschlossen.
Cyanobakterien (Blaualgen) unterscheiden sich von anderen Bakterien in ihrer Fähigkeit zur oxygenen Photosynthese. Diese findet in den Thylakoidmembranen in nahezu äquivalenter Weise wie in eukaryontischen Zellen statt. Neben Chlorophyll-a nutzen Cyanobakterien den Komplex Phycobilisom, in welchem die Phycobiline Phycocyanin und Phycoerythrin enthalten sind, zur Photosynthese. Diese Substanzen sind neben dem Chlorophyll für die Färbung der Zellen verantwortlich. Da die Zusammensetzung der Pigmente stark variiert, erscheinen Cyanobakterien blau, mitunter aber auch grün, rötlich oder schwarz.

Evolutionsbiologisch betrachtet sind Cyanobakterien die Vorläufer aller photosynthetisch aktiven Zellen. Vor etwa 3 Mrd. Jahren waren sie die ersten biologischen Sauerstoffproduzenten der Erde. Im Zuge ihrer Phylogenese besetzten sie nahezu alle ökologischen Nischen. Sie kommen in Gewässern, Böden, Wüsten, arktischen Regionen sowie in heißen Quellen vor. Unter entsprechenden Umweltbedingungen, wie eutrophem Gewässer, erhöhten Phosphat-, Nitrat- und Ammoniumkonzentrationen, bilden viele Arten enorme Massenansammlungen.

Viele Arten produzieren verschiedenste Sekundärmetabolite mit biologischer Aktivität. Die meisten dieser Substanzen sind Zellbestandteile und werden nur nach dem Zelltod freigesetzt. Oft sind sie toxisch und ursächlich für tödliche Vergiftungen bei Zuchtvieh, Wildtieren 
und sogar Menschen. Typische Symptome wie Hautreizungen, Bindehautentzündungen und Ohrenschmerzen treten sowohl nach oraler Aufnahme als auch nach dermatologischem Kontakt mit Cyanotoxinen belastetem Wasser auf. Darüber hinaus wurden Gastroenteritiden, Atemwegserkrankungen, Atemlähmung, allergische Reaktionen und Leberveränderungen festgestellt.

Die orale Aufnahme hat aus medizinischer Sicht die größte Bedeutung, weswegen vor allem Kleinkinder an Badestellen gefährdet sind. Je nach Konzentration der Cyanobakterien kann die letale Dosis für den Menschen bei wenigen $\mathrm{ml}$ Wasser liegen.

Die wichtigste Klasse der Cyanotoxine sind die Microcystine. Sie werden nur nach dem Tod der Produzenten in das Wasser abgegeben. Zu den wichtigsten Produzenten zählen die Arten Microcystis aeruginosa (Abb 1 a) und vor allem Planktothrix agardhii (Abb 1 b). Diese kommen vor allem im Süßwasser-Seen oder -Flüssen vor.

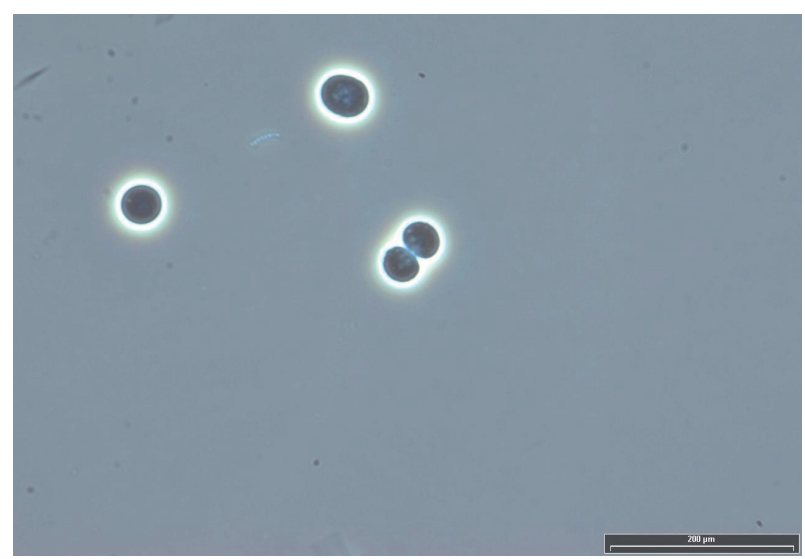

Abb. 1 a: Microcystis aeruginosa

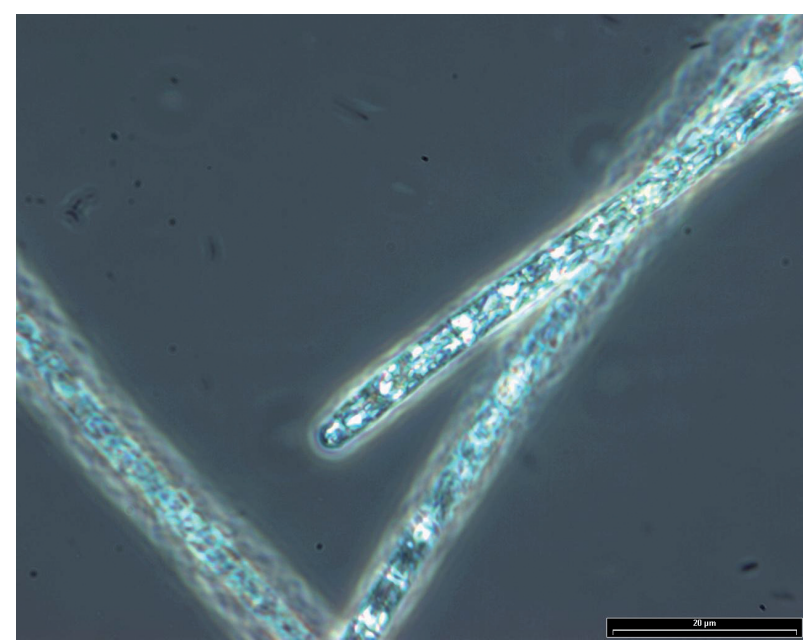

Abb. 1 b: Planktothrix agardhii

Microcystine sind zyklische Heptapeptide mit einem Molekulargewicht von 900 - 1100 g/mol. Fünf Aminosäuren dieser Peptidgruppe sind konstant und kommen nicht in Proteinen vor. Die zwei weiteren sind variabel und in Proteinen anzutreffen (Abb. 2). Der konservative Teil der Microcystine wird von den Aminosäuren D-Ala, D-Glu, D-erythro- $\beta$-Methyl-Asp, N-Methyl-dehydro-Ala und Adda (3-Amino-9-methoxy-2,6,8-trimethyl-10-phenyl4,6-decadien-carbonsäure) gebildet. Der variable Teil besteht aus 2 L-Aminosäuren. Es ist bekannt, dass die Microcystine nicht auf der DNA kodiert sind sondern verschiedene Enzymkomplexe deren Synthese katalysieren.

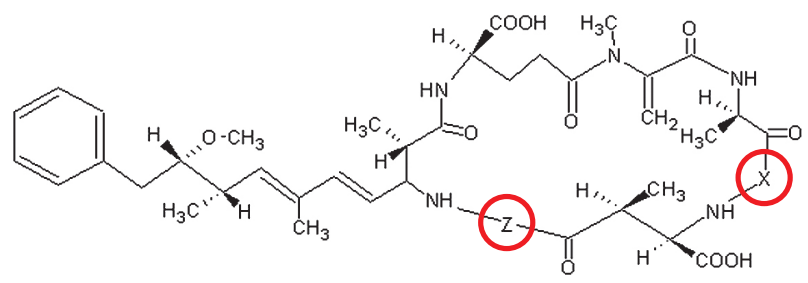

Abb. 2: Microcystine. Die variablen Bereiche sind $m i t ~ » X «$ und $» Z «$ gekennzeichnet.

Von den Microcystinen sind etwa 70 Varianten bekannt. Die toxikologisch bedeutendsten sind Microcystin-LR, -RR, und -LA. Sie bewirken akute Leberschädigungen (Erhöhung der Leberenzymkonzentration, Einblutungen, Nekrosen). Vergiftungen mit diesen Toxinen verursachen außerdem Schwäche, Lethargie, Bewegungsstörungen, Erbrechen und schließlich Kreislaufversagen.

Die Toxine werden über den Gallensäure-Carrier in die Zellen eingeschleust. Microcystine hemmen die Aktivität der Proteinphosphatasen 1 und 2A, welche die Zellteilung mitregulieren, durch irreversible, kovalente Bindung. Daher sind sie auch Tumorpromotoren.

Das Cyanotoxin Nodularin (Abb. 3) ist ein karzinogenes Pentapeptid mit einer molaren Masse von $824 \mathrm{~g} / \mathrm{mol}$. Die Eigenschaften von Nodularin und Microcystin sind beinahe identisch. Demzufolge sind auch die akuten und subakuten Symptome äquivalent. Nodularin wird von der Gattung Nodularina harveyana (Abb. 4) produziert.

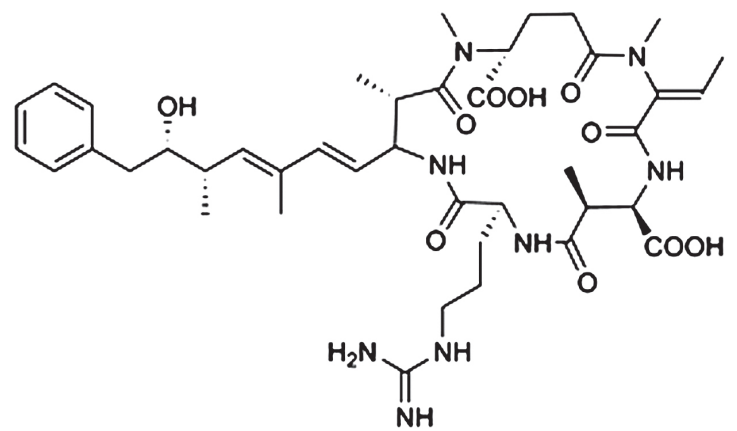

Abb. 3: Das Hepatotoxin Nodularin ist mit den Microcystinen strukturverwandt. Auch hier ist die charakteristische Aminosäure Adda vertreten.

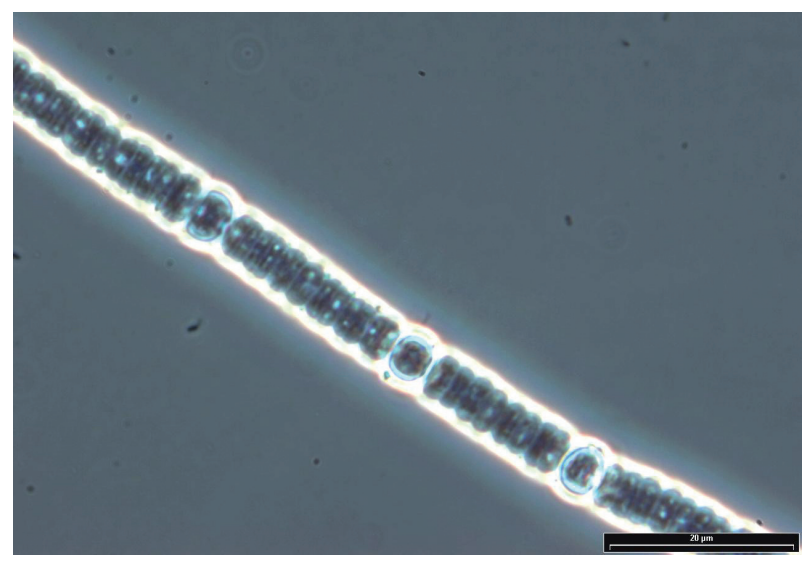

Abb. 4: Nodularia harveyana 
Das Gift Cylindrospermopsin (Abb. 5) wird u. a. von der Gattung Cylindrospermopsis raciborskii (Abb. 6) gebildet. In den letzten Jahren sind vermehrt Massenentwicklungen im Plattensee, in der Donau sowie in Brandenburgischen Gewässern beobachtet worden. Chemisch betrachtet ist Cylindrospermopsin ein tricyclisches Alkaloid, bestehend aus einem tricyclischen Guanidinrest kombiniert mit Hydroxymethyluracil (Abb. 5). Das Molekulargewicht beträgt $416 \mathrm{~g} / \mathrm{mol}$.

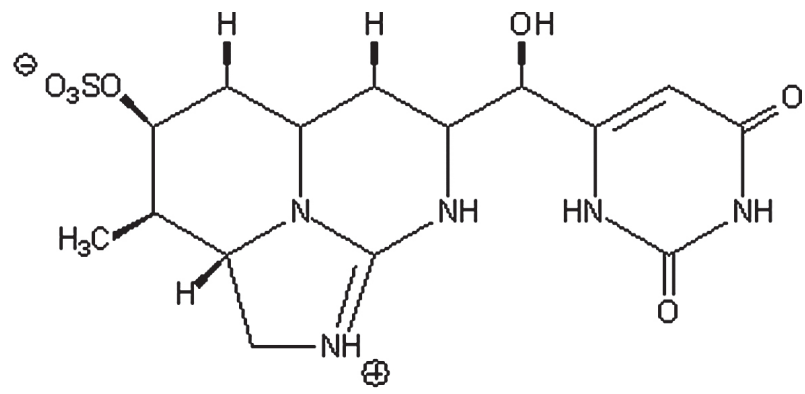

Abb. 5: Cylindrospermopsin

Die toxische Wirkung beruht auf der Hemmung der Eiweißsynthese. Dies ist besonders gefährlich, da die Hemmung kumulative Wirkungen zur Folge hat. Zu diesen zählen Hepatoenteritis, Hepatitis-ähnliche Symptome wie Unwohlsein, Anorexie, Erbrechen, Lebervergrößerung, Kopfschmerzen und Unterleibsschmerzen. Des Weiteren wurden Nierenversagen und blutige Diarrhö verzeichnet. Durch das Nierenversagen und den gastrointestinalen Ausfluss kam es in einigen Fällen zu starkem Elektrolytverlust, was Lethargie verursachte.

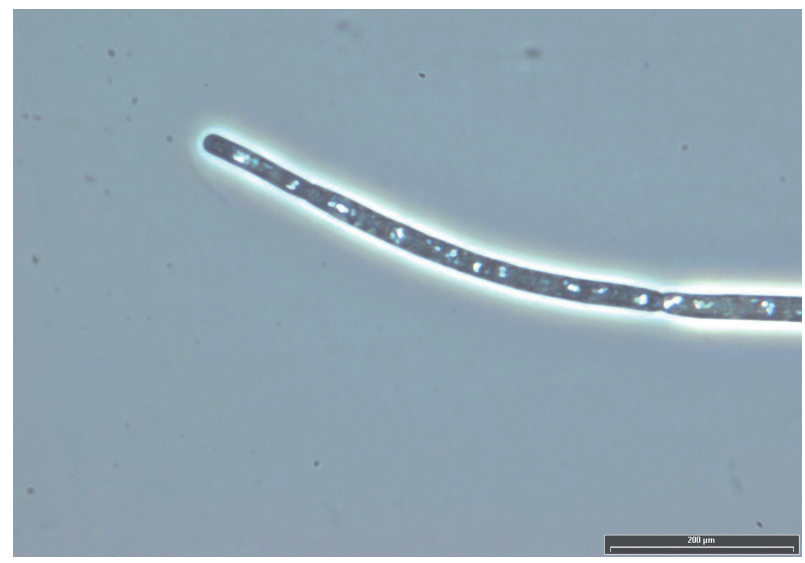

Abb. 6: Cylindrospermopsis raciborskii

Derzeitige Bestimmung der Cyanobakterientoxine Zunächst wird nur eine visuelle Inspektion des Gewässers vorgenommen. Treten Schlieren und Anschwemmungen auf und beträgt die Sichttiefe weniger als $1 \mathrm{~m}$, wird mikroskopisch auf Cyanobakterien geprüft.

Werden gefährliche Mengen von Cyanobakterien mikroskopisch bestätigt, wird anhand der Quantifizierung des Chlorophyll-a-Gehaltes das Biomassevolumen kalkuliert. Bei einer Konzentration $>40$ нg/l kann mit einer ähnlich hohen Konzentration Microcystin gerechnet werden.

Die Quantifizierung der Cyanotoxine erfolgt mit chemischen Analyseverfahren. Biochemische Nachweis- methoden sind nur für wenige Toxine verfügbar. Für die Extraktion der Toxine können verschiedene polare Lösemittel eingesetzt werden, 60 - 80 \% wässriges Methanol gilt aber als ideal, da es hydrophile und hydrophobe Toxine extrahiert.

Ein geeignetes Aufreinigungsverfahren ist die Festphasenextraktion mit reversed-phase C18-Materialien. Aber auch Gel- und Ionenaustauscherchromatographie sowie Immunoaffinitätsverfahren sind akzeptable Methoden.

Zur eigentlichen Toxindetektion haben sich der immunologische Nachweis (ELISA - Enzyme Linked Immuno Sorbent Assay) sowie die chromatographische Trennung mittels HPLC (High Performance Liquid Chromatography), mit anschließender UV-Detektion der Moleküle, etabliert.

Die Vorteile des immunologischen Nachweises sind seine einfache und relativ schnelle Durchführbarkeit sowie seine hohe Sensitivität von etwa $50 \mathrm{ng} / \mathrm{l}$. Nachteilig ist, dass nicht zwischen den Strukturvarianten der Microcystine unterschieden werden kann und Kreuzreaktionen auftreten können. Notwendig für die ELISADetektion ist der Einsatz spezifischer Antikörper. Diese werden vorwiegend über Immunisierungen von Tieren gewonnen. Allerdings sind solch kleine Moleküle wie die Toxine nur schwach oder nicht immunogen. Zudem stellt die Toxizität der Microcystine ein nicht zu vernachlässigendes Problem auch bei der Antikörper-Gewinnung dar. Im Rahmen der praktischen Durchführung werden die Zellen durch mehrmaliges Einfrieren und Auftauen aufgeschlossen, so dass die Toxine freigesetzt werden.

Mit Hilfe der HPLC lassen sich die Strukturvarianten der Microcystine unterscheiden. Allerdings ist dabei zwingend der Einsatz von Referenzsubstanzen erforderlich. Von den etwa 70 Microcystin-Strukturvarianten sind nur sehr wenige als Referenz erhältlich. Somit ist eine genaue Einschätzung der Toxizität der Probe noch nicht möglich, da diese innerhalb der Gifte variiert. Am häufigsten wird die Trennung über reversed-phase C18Säulen mit einem Gradienten, bestehend aus Wasser und Acetonitril, durchgeführt. Vorbereitend werden die Zellen aufgeschlossen und mehrfach mit wässrigem Methanol extrahiert. Nach zentrifugaler Klärung der Extrakte erfolgt die HPLC-Detektion.

Der Nachteil der bisher angewandten Verfahren ist der Zeitfaktor. Sowohl die immunologische als auch die chromatographische Detektion der Cyanobakterientoxine sind derzeit nur im Labor von entsprechendem Fachpersonal durchführbar. Bei beiden Verfahren liegen die Ergebnisse erst zwei Tage nach den Probenahmen vor. Des Weiteren stellt der Kostenfaktor in Zeiten der allgemeinen Haushaltlimitierungen ein nicht zu vernachlässigendes Problem dar. In Folge dieser Einschränkungen der Verfahren kann die neue Badegewässerrichtlinie nur unzureichend bzw. mit sehr hohem Kostenaufwand umgesetzt werden.

Ziel der an der Technischen Fachhochschule Wildau in Kooperation der Firma BioTeZ Berlin-Buch GmbH durchgeführten Forschungsarbeit ist es daher, ein Verfahren zu entwickeln, mit dem direkt am Gewässer auf 
Cyanobakterientoxine geprüft werden kann. Das System soll einfach und z. B. von einem Wassertechniker angewendet werden können. Die Analysenzeit soll sich im Bereich von 60 min befinden. Mit dem System sollen die Microcystine LA, RR, YR, LR, Cylindrospermopsin und Nodularin bestimmt werden. Dies leistet kein gegenwärtig verfügbares Messsystem.

\section{Methoden}

Um dieses anspruchsvolle Ziel zu erreichen, werden an der Technischen Fachhochschule Wildau sechs Gattungen Cyanobakterien kultiviert (Tab. 1). Diese sind Microcystis aeruginosa, Microcystis spec., Nodularia harveyana, Cylindrospermopsis raciborskii, Anabaena spec., Planktothrix agardhii. Aus diesen Spezies sollen dann die entsprechenden Cyanotoxine extrahiert werden, um den Assay zu entwickeln.

Für die Spezies, die von der SAG (Stammsammlung für Algenkulturen Göttingen) bezogen wurden, wurden verschiedene Medien angesetzt. Die Zusammensetzungen sind in den folgenden Tabellen dargestellt.

\begin{tabular}{|l|l|l|}
\hline Spezies & Produzierende Toxine & Medium \\
\hline Planktothrix agardhii & Microcystin-LA, -YR, -RR & ES-Medium \\
\hline Microcystis spec. & Microcystine & ES-Medium \\
\hline Anabaena spec. & Microcystine & $\begin{array}{l}1 / 2 \text { SWES- } \\
\text { Medium }\end{array}$ \\
\hline Nodularia harveyana & Nodularin & $\begin{array}{l}1 / 2 \text { SWES- } \\
\text { Medium }\end{array}$ \\
\hline Microcystis aeruginosa & Microcystine & Z-Medium \\
\hline $\begin{array}{l}\text { Cylindrospermopsis } \\
\text { raciborskii }\end{array}$ & Cylindrospermopsin & $\begin{array}{l}\text { WC-Medi- } \\
\text { um }\end{array}$ \\
\hline
\end{tabular}

Tab. 1: Übersicht der kultivierten Spezies, der Toxine und der Medien

\section{Zusammensetzung des ES-Mediums (Erddokat und Salze):}

\begin{tabular}{|c|c|c|c|}
\hline $\begin{array}{l}\text { Stamm- } \\
\text { lösungen }\end{array}$ & $\begin{array}{l}\text { Spurenelement- } \\
\text { Stammlösungen }\end{array}$ & $\begin{array}{l}\text { Spurenelement- } \\
\text { lösung }\end{array}$ & $\begin{array}{l}\text { gebrauchs- } \\
\text { fertiges } \\
\text { Medium }\end{array}$ \\
\hline $\begin{array}{l}10 \mathrm{~g} / \mathrm{l} \\
\mathrm{KNO}_{3}\end{array}$ & $1 \mathrm{~g} / \mathrm{ZnSO}_{4}$ & $\begin{array}{l}1 \mathrm{ml} \mathrm{ZnSO}_{4^{-}} \\
\text {Stammlösung }\end{array}$ & $\begin{array}{l}20 \mathrm{ml} \mathrm{KNO}_{3}^{-} \\
\text {Stammlösung }\end{array}$ \\
\hline $\begin{array}{l}1 \mathrm{~g} / \mathrm{l} \\
\mathrm{K}_{2} \mathrm{HPO}_{4}\end{array}$ & $0,76 \mathrm{~g} / \mathrm{l} \mathrm{MnSO}_{4}$ & 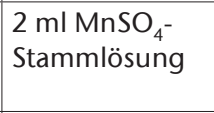 & $\begin{array}{l}20 \mathrm{ml} \\
\mathrm{K}_{2} \mathrm{HPO}_{4}^{-} \\
\text {Stammlösung }\end{array}$ \\
\hline \multirow[t]{7}{*}{$\begin{array}{l}1 \mathrm{~g} / \mathrm{l} \\
\mathrm{MgSO}_{4}\end{array}$} & $2 \mathrm{~g} / \mathrm{l} \mathrm{H}_{3} \mathrm{BO}_{3}$ & $\begin{array}{l}5 \mathrm{ml} \mathrm{H}_{3} \mathrm{BO}_{3^{-}} \\
\text {Stammlösung }\end{array}$ & $\begin{array}{l}20 \mathrm{ml} \mathrm{MgSO}_{4^{-}} \\
\text {Stammlösung }\end{array}$ \\
\hline & $0,2 \mathrm{~g} / \mathrm{Co}\left(\mathrm{NO}_{3}\right)_{2}$ & $\begin{array}{l}5 \mathrm{ml} \mathrm{Co}\left(\mathrm{NO}_{3}\right)_{2}^{-} \\
\text {Stammlösung }\end{array}$ & $\begin{array}{l}30 \mathrm{ml} \text { Boden- } \\
\text { extrakt }\end{array}$ \\
\hline & $0,2 \mathrm{~g} / \mathrm{l} \mathrm{Na} \mathrm{MoO}_{4}$ & $\begin{array}{l}5 \mathrm{ml} \mathrm{Na}_{2} \mathrm{MoO}_{4^{-}} \\
\text {Stammlösung }\end{array}$ & $\begin{array}{l}5 \mathrm{ml} \text { Spuren } \\
\text { element- } \\
\text { lösung }\end{array}$ \\
\hline & $0,005 \mathrm{~g} / \mathrm{l} \mathrm{CuSO}{ }_{4}$ & $\begin{array}{l}1 \mathrm{ml} \mathrm{CuSO}_{4^{-}} \\
\text {Stammlösung }\end{array}$ & $\begin{array}{l}905 \mathrm{ml} \\
\mathrm{ddH}_{2} \mathrm{O}\end{array}$ \\
\hline & & $0,8 \mathrm{~g} \mathrm{Na}_{2}$ EDTA & \\
\hline & & $0,7 \mathrm{~g} \mathrm{FeSO}_{4}$ & \\
\hline & & $\begin{array}{l}\text {-mit } \mathrm{ddH}_{2} \mathrm{O} \text { auf- } \\
\text { füllen auf } 1 \text { I }\end{array}$ & \\
\hline
\end{tabular}

Tab. 2: Zusammensetzung des ES-Mediums (nach SAG)
Der Bodenextrakt für das Medium wurde nach folgender Rezeptur hergestellt:

21 Erdboden über ein grobes Sieb (1 cm Maschenweite) sieben, anschließend feiner sieben (2 - 4 mm Maschenweite). Den gesiebten Boden in eine 5 1-Flasche

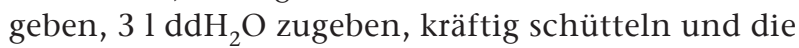
Suspension innerhalb von $24 \mathrm{~h}$ zwei mal für je $1 \mathrm{~h}$ bei $121^{\circ} \mathrm{C}$ autoklavieren. Die Suspension $10 \mathrm{~h}$ sedimentieren lassen. Den Überstand abdekantieren und über ein grobes Filter (Schleicher \& Schüll 597) filtrieren. Die Lösung dann bei $121^{\circ} \mathrm{C} 20 \mathrm{~min}$ autoklavieren.

\section{Zusammensetzung des $1 / 2$ SWES-Mediums}

( $1 / 2$ Seawater, Erddokat und Salze):

Die Zusammensetzung dieses Mediums ist der des ESMediums nahezu identisch. Der Unterschied besteht darin, dass bei diesem Medium zusätzlich 1,59\% (w/w) Meersalz hinzugefügt wurden.

\section{Zusammensetzung des Z-Mediums:}

Zunächst wurde eine Fe-EDTA-Lösung angesetzt. Dazu

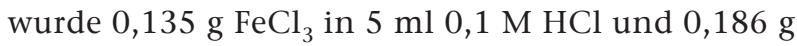
$\mathrm{Na}_{2}$ EDTA in $5 \mathrm{ml} \mathrm{ddH} \mathrm{H}_{2} \mathrm{O}$ gelöst. Beide Lösungen wurden vereint und auf $500 \mathrm{ml}$ aufgefüllt.

\begin{tabular}{|c|c|c|}
\hline $\begin{array}{l}\text { Stammlösungen } \\
\text { für Spuren- } \\
\text { elementlösung }\end{array}$ & Spurenelementlösung & $\begin{array}{l}\text { gebrauchsfertiges } \\
\text { Medium }\end{array}$ \\
\hline $\begin{array}{l}3,3 \mathrm{mg} / \mathrm{ml} \\
\mathrm{Na}_{2} \mathrm{WO}_{4}\end{array}$ & $1 \mathrm{ml} \mathrm{Na}_{2} \mathrm{WO}_{4}$-Stamm & $0,467 \mathrm{~g} / \mathrm{l} \mathrm{NaNO}$ \\
\hline $\begin{array}{l}8,8 \mathrm{mg} / \mathrm{ml} \\
\left(\mathrm{NH}_{4}\right)_{6} \mathrm{Mo}_{7} \mathrm{O}_{24}\end{array}$ & $\begin{array}{l}1 \mathrm{ml}\left(\mathrm{NH}_{4}\right)_{6} \mathrm{Mo}_{7} \mathrm{O}_{24^{-}} \\
\text {Stamm }\end{array}$ & $0,035 \mathrm{~g} / \mathrm{l} \mathrm{CaHPO}$ \\
\hline $11,9 \mathrm{mg} / \mathrm{ml} \mathrm{KBr}$ & $1 \mathrm{ml} \mathrm{KBr}-S t a m m$ & $0,031 \mathrm{~g} / \mathrm{I} \mathrm{K}_{2} \mathrm{HPO}_{4}$ \\
\hline $8,3 \mathrm{mg} / \mathrm{ml} \mathrm{KJ}$ & $1 \mathrm{ml} \mathrm{KJ-Stamm}$ & $0,025 \mathrm{~g} / \mathrm{l} \mathrm{MgSO}$ \\
\hline $\begin{array}{l}28,7 \mathrm{mg} / \mathrm{ml} \\
\mathrm{ZnSO}_{4}\end{array}$ & $1 \mathrm{ml} \mathrm{ZnSO}{ }_{4}-S_{\text {Stamm }}$ & $0,021 \mathrm{~g} / \mathrm{I} \mathrm{Na}_{2} \mathrm{CO}_{3}$ \\
\hline $\begin{array}{l}15,4 \mathrm{mg} / \mathrm{ml} \\
\mathrm{Cd}\left(\mathrm{NO}_{3}\right)_{2}\end{array}$ & $1 \mathrm{ml} \mathrm{Cd}\left(\mathrm{NO}_{3}\right)_{2}-$ Stamm & $\begin{array}{l}10 \mathrm{ml} \text { Fe-EDTA- } \\
\text { Lösung }\end{array}$ \\
\hline $\begin{array}{l}14,6 \mathrm{mg} / \mathrm{ml} \\
\mathrm{Co}\left(\mathrm{NO}_{3}\right)_{2}\end{array}$ & $1 \mathrm{ml} \mathrm{Co}\left(\mathrm{NO}_{3}\right)_{2}$-Stamm & $\begin{array}{l}\text { 0,080 ml Spuren- } \\
\text { elementlösung }\end{array}$ \\
\hline $\begin{array}{l}12,5 \mathrm{mg} / \mathrm{ml} \\
\mathrm{CuSO}_{4}\end{array}$ & $1 \mathrm{ml} \mathrm{CuSO}_{4}$-Stamm & $\begin{array}{l}\text { mit } \mathrm{ddH}_{2} \mathrm{O} \text { auf } 1 \mathrm{I} \\
\text { auffüllen }\end{array}$ \\
\hline $\begin{array}{l}19,8 \mathrm{mg} / \mathrm{ml} \\
\mathrm{NiSO}_{4}\left(\mathrm{NH}_{4}\right)_{2} \mathrm{SO}_{4}\end{array}$ & $\begin{array}{l}1 \mathrm{ml} \mathrm{NiSO}{ }_{4}\left(\mathrm{NH}_{4}\right)_{2} \mathrm{SO}_{4^{-}} \\
\text {Stamm }\end{array}$ & \\
\hline $\begin{array}{l}3,7 \mathrm{mg} / \mathrm{ml} \\
\mathrm{Cr}\left(\mathrm{NO}_{3}\right)_{3}\end{array}$ & $1 \mathrm{ml} \mathrm{Cr}\left(\mathrm{NO}_{3}\right)_{3}-$ Stamm & \\
\hline $2 \mathrm{mg} / \mathrm{ml} \mathrm{VOSO}_{4}$ & $1 \mathrm{ml} \mathrm{VOSO}_{4}$-Stamm & \\
\hline \multirow[t]{3}{*}{$\begin{array}{l}47,4 \mathrm{mg} / \mathrm{ml} \\
\mathrm{Al}_{2}\left(\mathrm{SO}_{4}\right)_{3} \mathrm{~K}_{2} \mathrm{SO}_{4}\end{array}$} & $\begin{array}{l}1 \mathrm{ml} \mathrm{Al}_{2}\left(\mathrm{SO}_{4}\right)_{3} \mathrm{~K}_{2} \mathrm{SO}_{4^{-}} \\
\text {Stamm }\end{array}$ & \\
\hline & $3,1 \mathrm{~g} / \mathrm{I} \mathrm{H}_{3} \mathrm{BO}_{3}$-Stamm & \\
\hline & $1,69 \mathrm{~g} / \mathrm{l} \mathrm{MnSO}{ }_{4}-$ Stamm & \\
\hline
\end{tabular}

Tab. 3: Zusammensetzung des Z-Mediums (nach SAG) 
Zusammensetzung des WC-Mediums

\begin{tabular}{|c|c|c|c|}
\hline $\begin{array}{l}\text { Stamm- } \\
\text { lösungen }\end{array}$ & $\begin{array}{l}\text { Spurenele- } \\
\text { mentlösung }\end{array}$ & Vitaminlösung & $\begin{array}{l}\text { gebrauchsfertiges } \\
\text { Medium }\end{array}$ \\
\hline $\begin{array}{l}27,9 \mathrm{~g} / \mathrm{l} \\
\mathrm{CaCl}_{2}\end{array}$ & $\begin{array}{l}4,36 \mathrm{~g} / \mathrm{l} \\
\mathrm{Na}_{2} \text { EDTA } \\
\end{array}$ & $\begin{array}{l}0,1 \mathrm{~g} / \mathrm{l} \\
\text { Thiamin- } \mathrm{HCl}\end{array}$ & $0,115 \mathrm{~g}$ TES \\
\hline $\begin{array}{l}37 \mathrm{~g} / \mathrm{l} \\
\mathrm{MgSO}_{4}\end{array}$ & $\begin{array}{l}3,15 \mathrm{~g} / \mathrm{l} \\
\mathrm{FeCl}_{3}\end{array}$ & $\begin{array}{l}0,0005 \mathrm{~g} / \mathrm{l} \\
\text { Biotin }\end{array}$ & $\begin{array}{l}1 \mathrm{ml} \mathrm{CaCl} 2^{-} \\
\text {Stammlösung }\end{array}$ \\
\hline $\begin{array}{l}12,6 \mathrm{~g} / \mathrm{l} \\
\mathrm{NaHCO}_{3}\end{array}$ & $\begin{array}{l}0,01 \mathrm{~g} / \mathrm{l} \\
\mathrm{CuSO}_{4}\end{array}$ & & $\begin{array}{l}1 \mathrm{ml} \mathrm{MgSO}_{4^{-}} \\
\text {Stammlösung }\end{array}$ \\
\hline $\begin{array}{l}8,75 \mathrm{~g} / \mathrm{l} \\
\mathrm{K}_{2} \mathrm{HPO}_{4}\end{array}$ & $\begin{array}{l}0,022 \mathrm{~g} / \mathrm{l} \\
\mathrm{ZnSO}_{4} \\
\end{array}$ & & $\begin{array}{l}1 \mathrm{ml} \mathrm{NaHCO}{ }^{-} \\
\text {Stammlösung }\end{array}$ \\
\hline $\begin{array}{l}85 \mathrm{~g} / \mathrm{l} \\
\mathrm{NaNO}_{3} \\
\end{array}$ & $\begin{array}{l}0,01 \mathrm{~g} / \mathrm{l} \\
\mathrm{CoCl}\end{array}$ & & $\begin{array}{l}1 \mathrm{ml} \mathrm{K}_{2} \mathrm{HPO}_{4}^{-} \\
\text {Stammlösung }\end{array}$ \\
\hline \multirow[t]{5}{*}{$21,2 \mathrm{~g} / \mathrm{l}$} & $\begin{array}{l}0,15 \mathrm{~g} / \mathrm{l} \\
\mathrm{MnCl}_{2}\end{array}$ & & $\begin{array}{l}1 \mathrm{ml} \mathrm{NaNO}_{3^{-}} \\
\text {Stammlösung }\end{array}$ \\
\hline & $\begin{array}{l}0,006 \mathrm{~g} / \mathrm{l} \\
\mathrm{Na}_{2} \mathrm{MoO}_{4} \\
\end{array}$ & & $\begin{array}{l}1 \mathrm{ml} \mathrm{Na}_{2} \mathrm{SiO}_{3}^{-} \\
\text {Stammlösung }\end{array}$ \\
\hline & $1 \mathrm{~g} / \mathrm{l} \mathrm{H}_{3} \mathrm{BO}_{3}$ & & $\begin{array}{l}1 \text { ml Spuren- } \\
\text { elementlösung }\end{array}$ \\
\hline & & & $1 \mathrm{ml}$ Vitaminlösung \\
\hline & & & $\begin{array}{l}\text { mit } \mathrm{ddH}_{2} \mathrm{O} \text { auf } 1 \mathrm{I} \\
\text { auffüllen }\end{array}$ \\
\hline
\end{tabular}

Tab. 4: Zusammensetzung des WC-Mediums (nach SAG)

Die Zellen wurden in den von der SAG vorgeschlagenen Medien kultiviert (Tab. 1). Zunächst wurden die Stämme in Zellkulturröhrchen kultiviert. Für jede Kultur wurden 4 Röhrchen angesetzt. In diese wurden je $15 \mathrm{ml}$ Medium steril eingefüllt und dazu $900 \mu l$ Zellsuspension gegeben. Das Animpfverhältnis betrug 1:17. Die Röhrchen wurden am Fenster und vor Leuchtstoffröhren untergebracht. Die Lampen emittieren das gesamte visuelle Lichtspektrum und sind hervorragend geeignet, um die Photosynthese der Zellen anzuregen. Das Lichtregime der Leuchtstoffröhren beträgt 12h/12h. Zwei mal täglich wurden die Röhrchen durchmischt. Die Kultivierung wird so lang durchgeführt, bis die Zellen die stationäre Phase erreichen.

Anschließend wurden die Kulturen in $200 \mathrm{ml}$ Erlenmeyerkolben überimpft, um die Biomasse zu erhöhen. Dazu wurde $100 \mathrm{ml}$ Medium in sterile Erlenmeyerkolben gegeben. Dazu wurde $8 \mathrm{ml}$ Zellsuspension pipettiert. Das Animpfverhältnis betrug 1:12,5. Die Kolben wurden auf einem Schüttler vor den Leuchtstoffröhren positioniert. Das Lichtregime betrug 12h/12h. Der Schüttler wurde auf 100 rpm eingestellt.

Zur Kultivierung der Zellen im größeren Maßstab wurde 1,5 1 des jeweiligen Mediums in 21 Schottflaschen gefüllt. Diese Flaschen wurden mit Laborverschraubungen versehen. Über diese wurde eine Probenahmestelle, eine Zuluftstelle und eine Abluftstelle montiert. Die Probenahmestelle wurde mit einer Schlauchklemme dicht verschlossen. Die Zuluft und Abluft wurden mit Sterilfiltern zur sterilen Begasung versehen. Die Flaschen wurden mit $100 \mathrm{ml}$ Zellsuspension angeimpft, das Animpfverhältnis betrug damit 1:15. Dies erfolgte steril. Zur Kultivierung wurden die Flaschen an eine Gasversorgungsanlage angeschlossen. Die Flaschen wurden mit Hilfe dieser mit Luft und $\mathrm{CO}_{2}$ (Air Liquide, $\mathrm{CO}_{2}$ 4.5 ) begast. Dabei wurden die Kulturen durchmischt (Abb. 7).

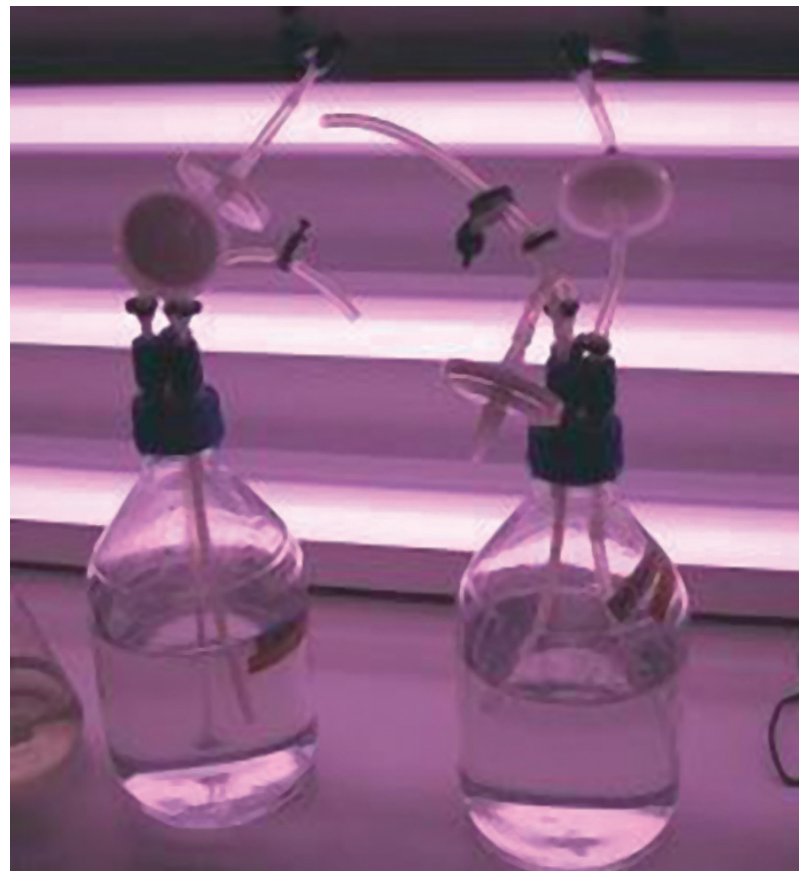

Abb. 7: Kultivierung der Zellen in 1,5 I Flaschen mit Luft- und $\mathrm{CO}_{2}-$ Begasung

Die Medien enthalten keine Zucker. Als Kohlenstoffquelle nutzen die Zellen Karbonate und das $\mathrm{CO}_{2}$ aus der Luft bzw. aus der Begasung. Dies kommt den natürlichen Bedingungen sehr nahe. Daneben wirkt das Medium limitierend und verringert das Risiko einer Kontamination durch Bakterien, die auf Zucker als Energiequelle angewiesen sind. Zur effizienteren Kultivierung wurden die 1,5 1 Schottflaschen neben Luft auch mit $\mathrm{CO}_{2}$ begast, um den Kohlenstoffgehalt im Medium zu erhöhen.

Zur Bestimmung der Zellzahl wurden verschiedene Verfahren getestet. Das einfache Zellzählen in einer Neubauer-Zählkammer erwies sich als unmöglich, da die Cyanobakterien zu stabilen Kolonien agglutinieren, die auch durch Behandlung im Ultraschallbad nicht aufgelöst werden können. Auch die einfache Trübungsmessung bei einer Wellenlänge von $600 \mathrm{~nm}$ erwies sich als ungeeignet. Der Grund hierfür liegt ebenso in der Agglutination der Zellen, die bewirkt, dass keine homogene Suspension vorliegt und somit die Messergebnisse verfälscht werden.

Aufgrund des Ausscheidens einfacher Verfahren wurde zur Kalkulation der Biomasse der Chlorophyll-aGehalt der Zellen bestimmt. Hierzu werden $20 \mathrm{ml}$ Zellsuspension entnommen und vakuumfiltriert. Das Filter und die darauf befindlichen Zellen wurden in Zentrifugationsröhrchen gegeben und es erfolgte eine 36-stündige Extraktion der löslichen Stoffe bei $20^{\circ} \mathrm{C}$ im Dunkeln mit Aceton. Danach wurden das Filter entnommen und die Röhrchen bei 3000 rpm für 15 Minuten zentrifugiert. Danach wurde die Chlorophyllmenge photometrisch bei 665 und $750 \mathrm{~nm}$ bestimmt. Die Messungen wurden so ausgeführt, dass es möglich war, sowohl die Menge Chlorophyll sowie die Menge Phäophytin zu bestimmen. An diesen Daten kann man erkennen, in welchem Stadium sich die Kultur befindet, da mit der Zeit der Anteil an Chlorophyll sinkt und der Anteil an Phäophytin steigt. 
Nach dem Erreichen der stationären Phase der Zellen in den 1,5 1 Flaschen sollen diese abgeerntet und aus ihnen die Toxine extrahiert werden. Im finalen Schritt dieses Aufreinigungsprozesses wird eine Bestimmung der Extrakte mittels HPLC vorgenommen. Dies ist nötig, um zu erfahren, ob und welche Toxine sich tatsächlich in den Extrakten befinden. Dazu ist es nötig, eine Kalibrierung durchzuführen. In Zuge dieser wurden und werden kommerzielle Cyanotoxine über die HPLC gegeben und detektiert. Die Trennung erfolgt in einer C18-Säule mit einer Durchflussrate von $0,5 \mathrm{ml} / \mathrm{min}$ und einer Säulentemperatur von $25^{\circ} \mathrm{C}$. Während der Trennung wird ein Lösemittel-Gradient mit dem in Tab. 5 gezeigten Gradienten gefahren.

\begin{tabular}{|l|l|l|}
\hline Zeit (min) & \% Acetonitril & $\%$ Wasser \\
\hline 0 & 50 & 50 \\
\hline 0 Bis 20 & 70 & 30 \\
\hline 20 Bis 25 & 100 & 0 \\
\hline 25 Bis 30 & 100 & 0 \\
\hline 30 Bis 45 & 50 & 50 \\
\hline 45 Bis 50 (Ende) & 50 & 50 \\
\hline
\end{tabular}

Tab. 5: Lösemittel-Gradient der HPLC

Die Detektion der Toxine erfolgte über UV-Messung bei $238 \mathrm{~nm}$.

\section{Ergebnisse}

Die Messung des Chlorophyll- und des PhäophytinWertes ergab, dass die Zellen im Erlenmeyerkolben besser bzw. schneller wachsen. Besonders die Gattungen Nodularia harveyana, Planktothrix agardhii und Cylindrospermopsis raciborskii, aber auch Anabaena spec. zeigen diesen Effekt (Abb. 8). Die weiteren zwei Gattungen zeigen keine signifikanten Differenzen zwischen den Kultivierungen im Erlenmeyerkolben und in den 1,5 1-Flaschen.

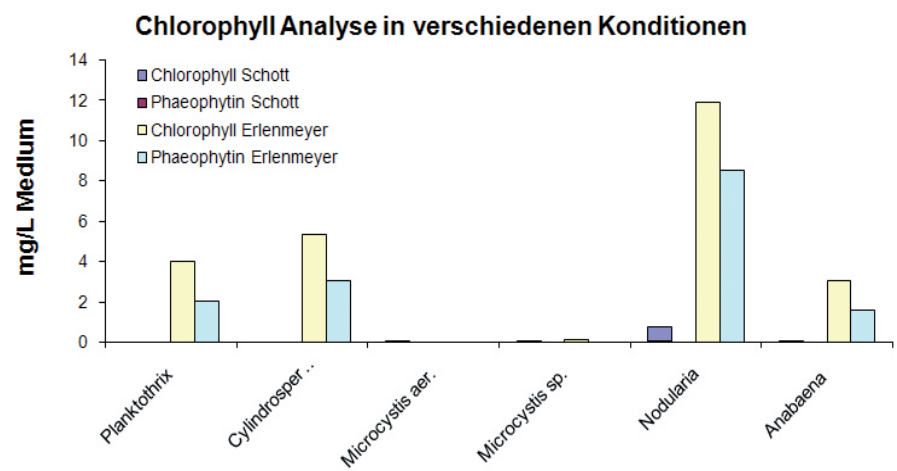

Abb. 8: Chlorophyll- und Phäophytin-Analyse der 6 kultivierten Spezies.

Eine generelle Aussage über die Potenz zur Kultivierung in den 1,5 l-Flaschen kann aber noch nicht getroffen werden, da sich die Experimente noch im Anfangsstadium befinden. In den Erlenmeyerkolben wurde wesentlich weniger Medium als in den Flaschen angesetzt. Demzufolge ist die Beleuchtungsintensität hier wesentlich höher als in den Flaschen. Daneben spielt der Gasaustausch eine nicht zu vernachlässigende Rolle. In den Kolben steht, gemessen am Volumen, eine sehr viel höhere Fläche für den Gasaustausch zu Verfügung. Womöglich ist die Begasung in den Flaschen zu ineffektiv, so dass das Gas nicht oder nur unzureichend in Lösung geht und deswegen nur die relativ kleine Grenzfläche zwischen Medium und Kopfraum zum Gasaustausch zur Verfügung steht. Diese Parameter könnten bewirken, dass die Zellen in den Flaschen sehr viel langsamer wachsen. Aus Zeitgründen konnte aber noch keine Langzeit-Kultivierung in den Schottflaschen vorgenommen werden, um die postulierten Vermutungen verifizieren zu können.

Das Ergebnis der HPLC-Messung zeigt einen eindeutigen Peak, der dem injizierten Microcystin-LR zugeordnet werden kann (Abb. 9).

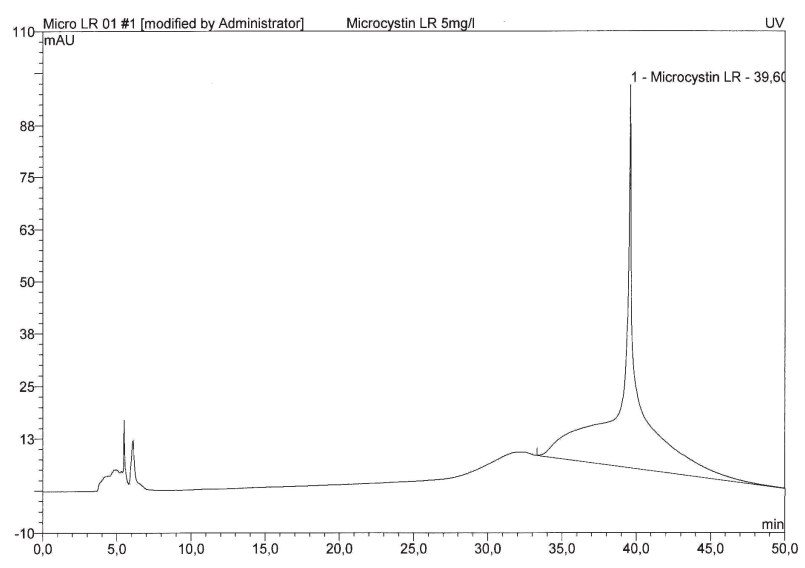

Abb. 9: HPLC-Messung von Microcystin- $L R$

Das Chromatogramm zeigt bei 39,6 min einen sehr hohen Peak, der eindeutig dem Microcystin-LR zugeordnet werden kann. Die Peaks im Bereich von 5 min können dem Methanol zugeordnet werden, da der MicrocystinLR-Standard in Methanol gelöst wurde.

Eine solche Kalibrierung wird für alle zu bestimmenden Cyanotoxine durchgeführt werden. Mit Hilfe dieser kann dann genau ermittelt werden, welche Toxine sich in den Extrakten befinden. Anhand der Peakfläche kann weiterführend auch ausgerechnet werden, in welcher Konzentration das jeweilige Toxin vorliegt.

\section{Ausblick}

Im weiteren Verlauf des Projektes werden die Zellen weiter so kultiviert, dass möglichst viel Biomasse gewonnen werden kann. Dies wird vermutlich in den 1,5 1 Flaschen geschehen. Folgend werden die Biomasse geerntet und die Toxine extrahiert. Die Extrakte werden mit der HPLC vermessen, um zu evaluieren, welche Toxine von den Zellen produziert worden sind.

Sobald diese Methoden stehen, wird nach einer Möglichkeit gesucht, die Zellen einfach aufzuschließen, um eine Vor-Ort-Toxin-Extraktionsmethode etablieren zu können. 


\section{Literatur}

Chorus, I./Bartram, J., Toxic Cyanobacteria in Water: A guide to their public health consequences, monitoring and management, WHO 1999

DGL - Deutsche Gesellschaft für Limnologie e.V., Tagungsbericht 1995

Hee-Mock, O. H. et al., Microcystin Production by Microcystis aeruginosa in a Phosphorus-Limited Chemostat, Applied and environmental Microbiology, 66, 176-179, 2000

Lawton, L. A., Extraction and High-performance LiquidChromatographic Method for the Determination of Microcystins in Raw and Treated Waters, Analyst, 119, 1525-1530, 1994

Pearl, H. W. et al., Adaptation to High-Intensity, Low-Wavelength Light among Surface Blooms of the Cyanobacterium Microcystis aeruginosa; Applied and environmental Microbiology; 49, 1046-1052, 1985

Richtlinie 2006/7/EG des Europäischen Parlaments und des Rates vom 15. Februar 2006 über die Qualität der Badegewässer und deren Bewirtschaftung und zur Aufhebung der Richtlinie 76/160/EWG, Artikel 8

Stanier, R. Y. et al., Purification and Properties of Unicellular Blue-Green Algae (Order Chroococcales), Bacteriological rewies, 35, 171-205, 1971

Watanabe, M./Oishi S., Effects of Environmental Factors on Toxicity of a Cyanobacterium (Microcystis aeruginosa) under Culture Conditions, Applied and environmental Microbiology, 49, 1342-1344, 1985

\section{Autoren}

Michél Hellmann, M. Sc.

Technische Fachhochschule Wildau

Biosystemtechnik/Bioinformatik

Tel. +493375 508-260

michel.hellmann@tfh-wildau.de

Judith Offermann, Ing.

Instituto Politécnico de Brangança

Portugal

Prof. Dr. rer. nat. Franz X. Wildenauer

Technische Fachhochschule Wildau

Biosystemtechnik/Bioinformatik

Tel. +493375 508-148

franz-xaver.wildenauer@tfh-wildau.de 